PAPER

\title{
Alzheimer's disease-one clinical syndrome, two radiological expressions: a study on blood pressure
}

\author{
F-E de Leeuw, F Barkhof, P Scheltens
}

J Neurol Neurosurg Psychiatry 2004;75:1270-1274. doi: 10.1136/jnnp.2003.030189

See end of article for authors' affiliations .....................

Correspondence to: Professor Philip Scheltens, Alzheimer Centre, Department of Neurology, VU Medical Centre, PO Box 7057, 1007MB Amsterdam, Netherlands; p.scheltens@vumc.nl

Received 10 October 2003 Revised 7 December 2003 Accepted 17 December 2003

\begin{abstract}
Background: Vascular risk factors could play a role in the aetiology of Alzheimer's disease, but this has not been investigated in relation to neuroimaging findings

Objective: To evaluate the distribution of blood pressure and an indicator of atherosclerosis (pulse pressure) in patients with Alzheimer's disease with and without small vessel disease.

Methods: 152 Alzheimer patients underwent 1.0T MRI scanning. Blood pressure was measured with a sphygmomanometer. Small vessel disease was assessed by the presence of lacunar infarcts and white matter lesions. The distribution of blood pressure and pulse pressure, with or without small vessel disease, was assessed by linear regression analysis.

Results: Patients with small vessel disease had a higher blood pressure, a wider pulse pressure, and an increased prevalence of hypertension. These findings were strongly age dependent: for patients under 65 , mean systolic blood pressure was higher in the subpopulation with small vessel disease than in those without (mean (SD): 149.9 (19.3) v 135.7 (20.5) mm Hg; $p=0.02$ ). Hypertension was more common in patients with white matter lesions than in those without $(75.6 \% \vee 45.1 \% ; p=0.03)$ and the pulse pressure was higher (61.9 (14.4) v $51.7(11.5) \mathrm{mm} \mathrm{Hg} ; \mathrm{p}=0.01)$. There was no relation between blood pressure and the degree of (sub)cortical and hippocampal atrophy in patients without small vessel disease.

Conclusions: There was heterogeneity in Alzheimer's disease patients with respect to blood pressure and pulse pressure. Alzheimer's disease encompasses a heterogeneous group of disorders which share a common cognitive profile but with distinct radiological features with respect to white matter lesions.
\end{abstract}

disease would have a substantially higher blood pressure and pulse pressure than those without.

\section{METHODS}

\section{Study population}

Subjects were selected from individuals who were consecutively investigated for suspected dementia at the secondary/ tertiary referral Alzheimer Centre at the Vrije Universiteit Medical Centre, Amsterdam, Netherlands. We identified 152 consecutive subjects who had complete data on a the standardised work-up in a five year period which involved history taking, physical and neurological examination, blood tests (erythrocyte sedimentation rate, haemoglobin, white cell count, serum electrolytes, glucose, creatinine, liver function tests, thyroid stimulation hormone and free thyroid hormone, vitamin B-1 and B-6 levels, and syphilis serology), mini-mental state examination (MMSE), comprehensive neuropsychological examination, MRI of the brain, and quantitative electroencephalography. The final diagnosis was based on a consensus meeting where all the available clinical data and the results of the ancillary investigations were reviewed. A diagnosis of probable Alzheimer's disease was based upon the NINCDS-ADRDA criteria. ${ }^{1}$ We only included in the study those patients in whom the diagnosis remained unchanged after a minimum follow up period of one year. All patients provided written informed consent for their clinical data being used for research.

Abbreviations: MMSE, mini-mental state examination; MTA, medial temporal lobe atrophy; NINCDS-ADRDA, National Institute of Neurological and Communicative Disorders and Stroke and Alzheimer's disease and Related Disorders Association among Alzheimer patients with and without small vessel disease. We hypothesised that patients with small vessel 


\section{Measurements}

Blood pressure was measured manually in a standardised manner using a sphygmomanometer, with the patient in sitting position after five minutes of rest. Values were based on a single measurement. The first and the fourth Korotkoff sounds were used for the systolic and diastolic blood pressure, respectively. Hypertension was defined as a systolic blood pressure of $\geqslant 140 \mathrm{~mm} \mathrm{Hg}$ or a diastolic blood pressure of $\geqslant 90 \mathrm{~mm} \mathrm{Hg}$ or both, with or without the use of blood pressure lowering drugs. Pulse pressure was calculated as the difference between systolic and diastolic blood pressure. It is related to arterial stiffness and as such represents a measure of atherosclerosis. ${ }^{13}$ The mean time interval between blood pressure measurement and MRI scanning was about six months.

Smoking status (current/former) and drug history was obtained from the patients' medical notes. Diabetes mellitus was considered present if the participant was taking glucose lowering drugs or if a random blood glucose was above 11.1 $\mathrm{mmol} / \mathrm{l}$.

Cognitive function was assessed by administering the mini-mental state examination (MMSE) at the first outpatient clinic appointment, to establish the severity of global dementia. ${ }^{14}$

\section{MRI scanning protocol}

All subjects underwent cranial MRI including coronal Tl weighted and transverse proton density or fluid attenuated inversion recovery (FLAIR) images on a 1.0T scanner (Impact, Siemens AG, Erlangen, Germany) using a standardised protocol, including contiguous $3 \mathrm{~mm}$ thick slices for the $\mathrm{Tl}$ coronal images and $5 \mathrm{~mm}$ thick slices with an interslice gap of $20.0 \%$ for the proton density and FLAIR images. All sequences yielded an in-plane resolution of $1 \times 1 \mathrm{~mm}^{2}$.

\section{Small vessel disease: white matter lesions and lacunar infarcts}

White matter lesions were rated using the Rotterdam scan study rating scale. ${ }^{8}$ The rater was blinded to any clinical information about the patient. White matter lesions were considered present if these were hyperintense on proton density or FLAIR images without prominent hypointensity on Tl weighted images. White matter lesions were assessed according to location in subcortical and periventricular regions using a previously described protocol ${ }^{8}$ : in short, the number and size of subcortical white matter lesions was rated on hard copy according to their largest diameter in categories of small $(<3 \mathrm{~mm})$, medium $(3-10 \mathrm{~mm})$, or large lesions $(>10 \mathrm{~mm})$; periventricular white matter lesions were rated semiquantitatively per region: adjacent to frontal horns (frontal capping); adjacent to lateral wall of lateral ventricles (bands), and adjacent to occipital horns (occipital capping), on a scale ranging from 0 (no white matter lesions), to 1 (pencil thin periventricular lining), 2 (smooth halo or thick lining), or 3 (large confluent white matter lesions). The overall degree of periventricular white matter lesions was calculated by adding up the scores for the three separate regions (range 0 to 9 ).

Lacunar infarcts were defined as focal hyperintensities smaller than $15 \mathrm{~mm}$ on T2 weighted images, with a corresponding hypointensity on a Tl weighted image..$^{15}$

\section{Cerebral atrophy}

Subcortical atrophy was assessed using the ventricle to brain ratio (mean of the biventricular width at the level of the frontal and occipital horns and at the level of the caudate nuclei, divided by the corresponding brain width at these levels). ${ }^{17}$
Cortical atrophy was assessed as the mean of the maximum width of the left and right Sylvian fissure divided by the maximum (transpineal) brain width. ${ }^{17}$

Medial temporal lobe atrophy (MTA) was assessed by means of a previously reported visual rating scale. In short, MTA was rated on a coronal Tl weighted image based on the width of the choroid fissure and the temporal horn and the height of the hippocampal formation, resulting in a score ranging from 0 (normal appearance of any of these three variables) to 4 (severe widening of the choroid fissure and the temporal horn and a reduced hippocampal height). ${ }^{18}$

Intrarater $\kappa$ values for periventricular white matter lesion severity grades were between 0.6 and 0.8 . The intrarater, intraclass correlation coefficient for subcortical white matter rating was 0.95 . The intrarater, intraclass correlation coefficients for subcortical and cortical atrophy were 0.7 to 0.8 and 0.6 , respectively.

\section{Statistical analysis}

The relation between vascular factors and the presence of small vessel disease was assessed by linear regression analysis adjusted for age (at MRI scanning) and sex. Small vessel disease was defined as present if there was a single white matter lesion or lacunar infarct, irrespective of its size or location; otherwise it was coded as absent.

We calculated the mean systolic and diastolic blood pressure, pulse pressure, and the prevalence of hypertension in Alzheimer patients with or without small vessel disease by means of age and sex adjusted linear regression. Additional adjustments were made for smoking, diabetes, and MMSE

The relation between blood pressure and white matter lesions may be biased by selective survival, as the level of blood pressure is related to mortality. To address this issue we carried out an age stratified analysis because we expected a less pronounced effect of this selective mortality in the youngest age category. ${ }^{9}$ Although the cross sectional design of our study does not allow the formal testing of causality between blood pressure and small vessel disease, we did assess the relation between the level of blood pressure and the degree of white matter lesions in patients with small vessel disease.

Merely assessing blood pressure levels in patients without small vessel disease does not preclude a causal relation between blood pressure and Alzheimer's disease in this subpopulation. It could be that blood pressure increases the risk for Alzheimer's disease by inducing changes other than structural small vessel disease, such as hippocampal, subcortical, or cortical atrophy. We therefore investigated the relation between blood pressure and these structural changes in patients without small vessel disease using age and sex adjusted multiple linear regression analysis.

\section{RESULTS}

Table 1 presents the baseline characteristics of the study population. Patients with Alzheimer's disease plus small vessel disease were older than those without small vessel disease (mean (SD): 70.0 (8.3) v 63.5 (8.5) years, $\mathrm{p}<0.01$ ). In both subpopulations there were slightly more women than men, but the difference was not statistically significant. Of all participants, $23.6 \%(n=36)$ had no signs of small vessel disease on MRI. There were seven patients with a lacunar infarct, one of whom had no white matter lesions. The median severity of periventricular white matter lesions was 1.0 (1.8) and the mean number of subcortical white matter lesions, 55.2 (118.4). Dementia severity was comparable within the two groups (mean MMSE, 21.0 (5.8) with small vessel disease $v 20.6$ (5.1) without small vessel disease). There was no difference in hippocampal, subcortical, or cortical atrophy between the two subpopulations. 
Table 1 Characteristics of the study population by presence or absence of small vessel disease*

\begin{tabular}{lll}
\hline & \multicolumn{2}{l}{ Small vessel disease } \\
\cline { 2 - 3 } Characteristic & Absent & Present \\
\hline $\mathrm{n}$ & 36 & 116 \\
Women (\%) & 54.3 & 51.9 \\
Mean age (years) & $63.5(8.3)$ & $70.0(8.3) \dagger$ \\
$\quad<65$ years (n) & 19 & 31 \\
$\quad \geqslant 65$ years ( $\mathrm{n})$ & 17 & 85 \\
Diabetes mellitus (\%) & 13.5 & 3.5 \\
Ever smoked (\%) & 27.9 & 41.2 \\
MMSE & $21.0(5.8)$ & $20.6(5.1)$ \\
MTA & $1.5(1.0)$ & $1.7(1.0)$ \\
Subcortical atrophy & $0.34(0.04)$ & $0.35(0.04)$ \\
Cortical atrophy & $0.1(0.03)$ & $0.1(0.04)$ \\
\hline
\end{tabular}

*Values are age and sex adjusted means (SD) or percentages.

Differences between groups were tested by age and sex adjusted analysis of covariance.

$\mathrm{tp}<0.01$.

MMSE, mini-mental state examination; MTA, medial temporal atrophy

Table 2 presents the mean blood pressure in the two subpopulations. For participants aged below 65 years, the mean systolic blood pressure was significantly higher in subjects with small vessel disease than in those without ( 149.9 (19.3) v 135.7 (20.5) $\mathrm{mm} \mathrm{Hg;}=0.02$ ). They also had a higher diastolic blood pressure, although this did not reach statistical difference. In addition, the prevalence of hypertension was greater than in subjects without white matter lesions $(75.6 \%$ v 45.1\%; $p=0.03)$. Alzheimer patients under the age of 65 years with white matter lesions also had a significantly higher pulse pressure (61.9 (14.4) v 51.7 (11.5) $\mathrm{mm} \mathrm{Hg} ; \mathrm{p}=0.01)$. This effect was strongly associated with increasing age. Adjustments for the possible confounding factors did not have a substantial effect on the outcome of the analysis.

Within the subpopulation of Alzheimer patients with small vessel disease there was a relation between both systolic and diastolic blood pressure and the extent of the periventricular white matter lesions $\left(\mathrm{p}_{\text {trend }}=0.055\right.$ and 0.056 , respectively; fig 1). Patients with hypertension had more severe periventricular white matter lesions in that same stratum (mean degree, $1.5(1.6) \vee 2.3(2.2) ; \mathrm{p}=0.06)$. There was no such relation for subcortical white matter lesions (fig 2).

Within the Alzheimer patient subpopulation without small vessel disease, there was no relation between blood pressure or hypertension and the degree of (sub)cortical and hippocampal atrophy.

\section{DISCUSSION}

We studied the distribution of blood pressure and an indicator of atherosclerosis (pulse pressure) in patients with Alzheimer's disease with and without small vessel disease. We found that both the systolic and diastolic blood pressure and the prevalence of hypertension were higher among patients with small vessel disease than among those without. An identical observation was made for pulse pressure. The groups did not differ with respect to other MRI measures or MMSE.

Among the strengths of our study were the large number of community dwelling Alzheimer patients who all underwent cerebral MRI scanning using a standardised protocol, the length of follow up, and the use of a single blinded scan rater with a high intrarater consistency. Some methodological issues, however, need to be addressed. Our results may in part be biased owing to selective survival. ${ }^{19}$ When studying a risk factor for small vessel disease that is related to mortality it seems plausible that increased mortality may occur particularly among patients with the longest duration of the disease-that is, generally the oldest patients. ${ }^{19}$ As in previous studies, ${ }^{9}$ we addressed this issue by carrying out an age stratified analysis, because hypertension related mortality plays a less important role in the youngest age category. Consequently, the blood pressures measured in the youngest category represent the most unbiased values.

Selection bias could also occur because blood pressure may be related to the accessibility of the outpatient clinic, which will vary depending on the presence of gait disturbance. ${ }^{20}$ This form of bias will therefore underestimate the blood pressure levels in the group with small vessel disease, as those with the highest blood pressure are not represented.

Misclassification of blood pressure could have played a role because previously high blood pressure levels tend to decrease once Alzheimer's disease has been diagnosed. ${ }^{21}$ Unfortunately we did not have blood pressure measurements

Table 2 Mean systolic and diastolic blood pressure, prevalence of hypertension, and pulse pressure by absence or presence of small vessel disease in patients with Alzheimer's disease $^{*}$

\begin{tabular}{|c|c|c|c|}
\hline & \multirow[b]{2}{*}{ Age at MRI } & \multicolumn{2}{|c|}{ Small vessel disease } \\
\hline & & Absent $(n=36)$ & Present $(n=116)$ \\
\hline Systolic blood pressure & $\begin{array}{l}<65 \text { years }(n=50) \\
\geqslant 65 \text { years }(n=102) \\
\text { Overall }(n=152)\end{array}$ & $\begin{array}{l}135.7(20.5) \\
156.5(21.0) \\
146.9(22.2)\end{array}$ & $\begin{array}{l}149.9(19.3) \dagger \\
151.9(23.1) \\
150.9(22.1)\end{array}$ \\
\hline Diastolic blood pressure & $\begin{array}{l}<65 \text { years } \\
\geqslant 65 \text { years } \\
\text { Overall }\end{array}$ & $\begin{array}{l}84.0(11.6) \\
86.3(8.8) \\
85.2(10.2)\end{array}$ & $\begin{array}{l}88.0(12.1) \\
86.2(11.1) \\
86.7(11.4)\end{array}$ \\
\hline Hypertension & $\begin{array}{l}<65 \text { years } \\
\geqslant 65 \text { years } \\
\text { Overall }\end{array}$ & $\begin{array}{l}45.1 \\
92.6 \\
71.6\end{array}$ & $\begin{array}{l}75.6 \ddagger \\
80.3 \\
77.8\end{array}$ \\
\hline Pulse pressure & $\begin{array}{l}<65 \text { years } \\
\geqslant 65 \text { years } \\
\text { Overall }\end{array}$ & $\begin{array}{l}51.7(11.5) \\
70.1(16.4) \\
61.7(16.1)\end{array}$ & $\begin{array}{l}61.9(14.4) \\
65.7(18.7) \\
64.3(17.7)\end{array}$ \\
\hline
\end{tabular}

*Values are age and sex adjusted means (SD) or percentages. Differences between those with or without small vessel disease were tested by age and sex adjusted analysis of covariance. $\dagger p=0.02 ; \neq p=0.03 ; \uparrow p=0.01$.

MRI, magnetic resonance imaging 


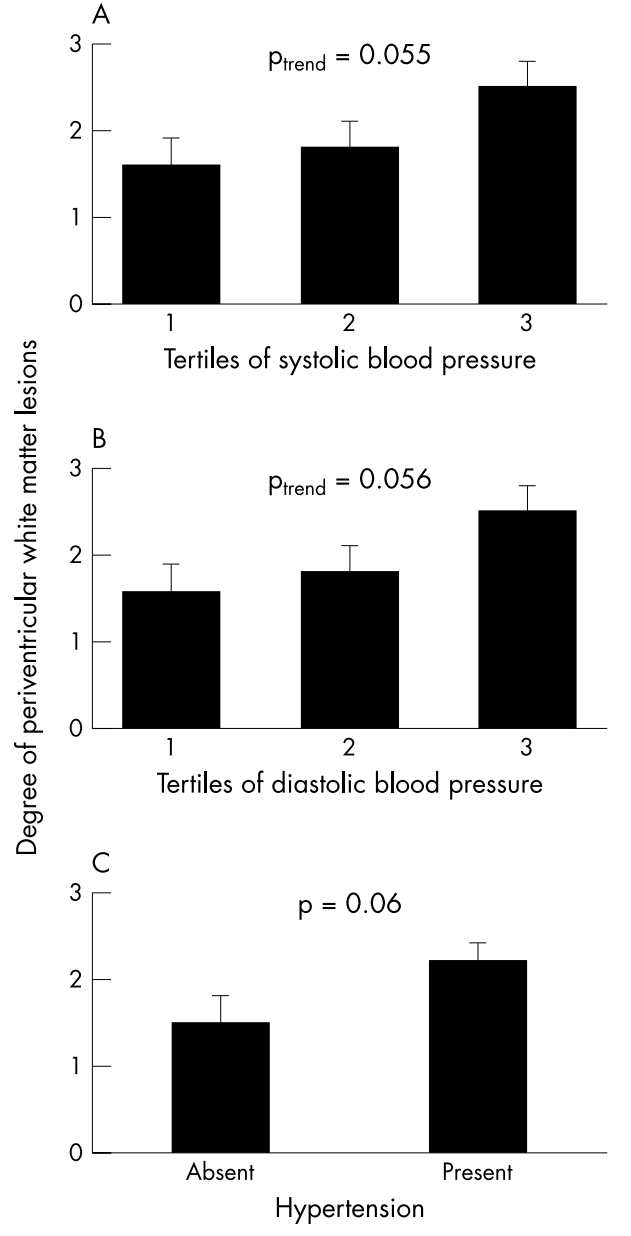

Figure 1 Degree of periventricular white matter lesions by tertiles of systolic blood pressure (A) and diastolic blood pressure (B). (C) Degree of periventricular white matter lesions in patients with or without hypertension. Values are means, error bars $=$ SEM.

before the onset of the disease. As all patients in our study suffered from Alzheimer's disease, this potential misclassification would have affected all patients equally, probably without having an effect on the difference in blood pressure levels between those with and without small vessel disease.

Our finding of blood pressure differences between Alzheimer patients with and without small vessel disease may be confounded by other vascular risk factors, including smoking, diabetes, and cholesterol. However, adjustments for smoking and diabetes did not alter the blood pressure differences between the groups, suggesting an independent effect. Unfortunately, no information on cholesterol was available in our study. A recent randomised trial found no effect of cholesterol lowering on the incidence of Alzheimer's disease, $^{22}$ and we think it unlikely that cholesterol acts as a confounder for blood pressure in our study.

Our study was cross sectional and we therefore cannot exclude a relation between vascular risk factors and the incidence of Alzheimer's disease in patients without small vessel disease. An argument against this notion is that we found no relation between blood pressure, hypertension, and Alzheimer's disease related cerebral changes, including (sub)cortical or hippocampal atrophy, in this subpopulation.

Our study provides evidence for heterogeneity in Alzheimer's disease in the distribution of blood pressure within a population with clinically diagnosed Alzheimer's disease with or without small vessel disease. Vascular risk
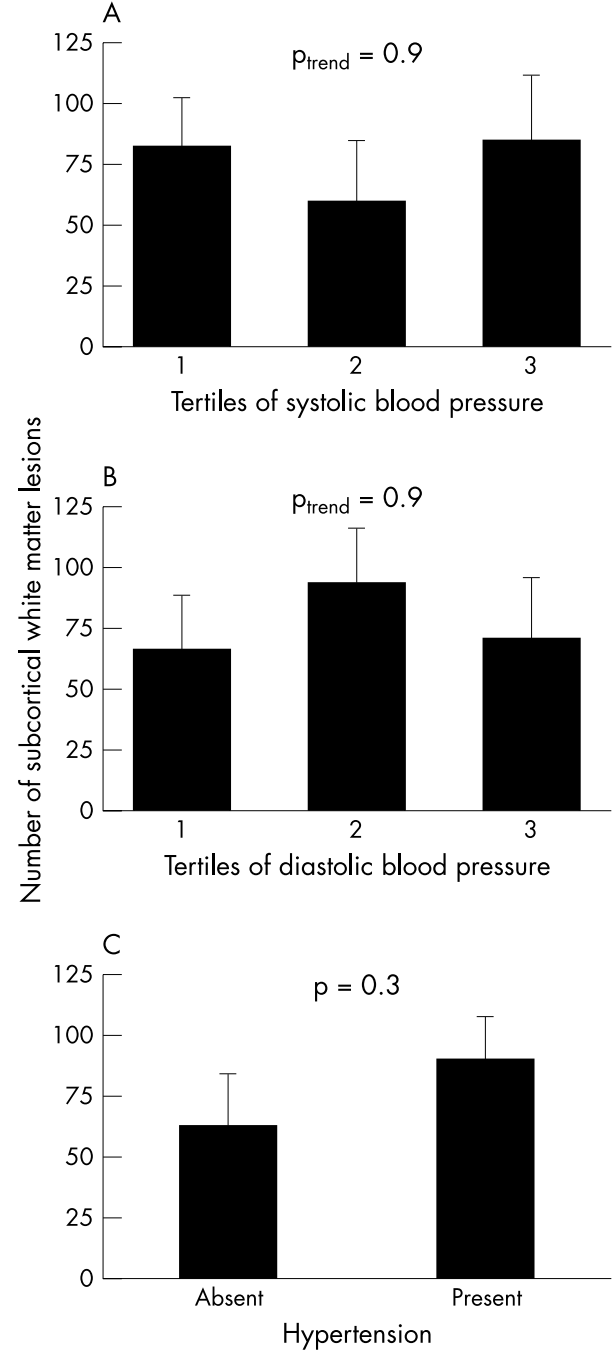

Figure 2 Number of subcortical white matter lesions by tertiles of systolic blood pressure (A) and diastolic blood pressure (B). (C) Number of subcortical white matter lesions in patients with or without hypertension. Values are means, error bars $=S E M$.

factors including hypertension and indicators of atherosclerosis are established risk factors for the presence of small vessel disease ${ }^{7-9}$ and in the subpopulation of Alzheimer's disease with small vessel disease those factors may be related to their presence. ${ }^{16}$ Our finding of a linear relation between blood pressure and the severity of white matter lesions in the Alzheimer group with small vessel disease supports this view. Previous studies showed a relation between the degree of white matter lesions and cognitive impairment. ${ }^{23-25}$ It is therefore possible that these vascular factors, acting through the emergence of white matter lesions or lacunar infarcts, are at least in part responsible for the cognitive impairment in these Alzheimer patients. However, we do not have a standardised neuropsychological examination of each Alzheimer patient in order to assess the relation between white matter lesions and cognitive function.

The notion of radiological heterogeneity in Alzheimer's disease $^{26}$ is compatible with the observed neuropathological heterogeneity of this disorder. Two neuropathological subtypes of Alzheimer's disease are recognised, one with predominantly plaques and tangles ${ }^{27} 28$ (without the presence of co-pathology), and a second subtype with additional pathological changes including age related neuronal loss, lacunar infarcts, and white matter lesions. ${ }^{29}$ Notably, the 
patients from the first group appear to be younger, which is in agreement with our finding of a significant younger age in the group without white matter lesions. Unfortunately we do not have neuropathology for our cases in order to compare radiological and neuropathological findings.

There is a growing awareness of vascular risk factors in the aetiology of Alzheimer's disease. This is supported by data from the double blind randomised Syst-Eur trial in which patients treated for isolated systolic hypertension had a significantly reduced incidence of Alzheimer's disease. ${ }^{30}$ However, in those studies Alzheimer patients were included on the basis of the clinical NINCDS-ADRA criteria without taking into account small vessel disease on neuroimaging. In view of our findings it is possible that treatment of hypertension will only benefit the subpopulation of Alzheimer patients with small vessel disease by influencing the occurrence of new small vessel disease, or possibly even by halting the progression of existing lesions. The latter hypothesis requires testing in a clinical trial on Alzheimer patients using MRI.

\section{Conclusions}

We have found evidence for heterogeneity with respect to blood pressure in patients with Alzheimer's disease. A substantial proportion of clinically diagnosed cases do not have any signs of small vessel disease. Our findings call for a reassessment of the relation between vascular risk factors and Alzheimer's disease and may provide support for subclassifying Alzheimer's disease on the basis of the presence or absence of small vessel disease. This may benefit not only epidemiological research into risk factors but also the development of new treatment strategies.

\section{ACKNOWLEDGEMENTS}

F-EDL's stay at the Alzheimer Centre VU was supported financially by the Stichting Alzheimer and Neuropsychiatrie, Amsterdam. Dr John Janssen MRCP kindly reviewed the manuscript for English style and grammar.

\section{Authors' affiliations}

F-E de Leeuw, Department of Neurology, University Medical Centre Niimegen, Netherlands

P Scheltens, Alzheimer Centre and Department of Neurology, VU Medical Centre, Amsterdam, Netherlands

F Barkhof, Department of Radiology, VU Medical Centre, Amsterdam Competing interests: none declared

\section{REFERENCES}

1 McKhann G, Drachman D, Folstein M, et al. Clinical diagnosis of Alzheimer's disease: report of the NINCDS-ADRDA Work Group under the auspices of Department of Health and Human Services Task F on Alzheimer's Disease. Neurology 1984;34:939-44.

2 Etiene D, Kraft J, Ganju N, et al. Cerebrovascular pathology contributes to the heterogeneity of Alzheimer's disease. J Alzheimers Dis 1998;1:119-34.

3 Doddy RS, Massman PJ, Mawad M, et al. Cognitive consequences of subcortical magnetic resonance imaging changes in Alzheimer's disease: comparison to small vessel ischemic vascular dementia. Neuropsychiatry Neuropsychol Behav Neurol 1998;1 1:191-9.

4 Lopez OL, Becker JT, Jungreis CA, et al. Computed tomography - but not magnetic resonance imaging - identified periventricular white-matter lesions predict symptomatic cerebrovascular disease in probable Alzheimer's disease. Arch Neurol 1995;52:659-64

5 DeCarli C, Grady CL, Clark CM, et al. Comparison of positron emission tomography, cognition, and brain volume in Alzheimer's disease with and without severe abnormalities of white matter. I Neurol Neurosurg Psychiatry 1996;60:158-67.

6 van Gool WA, Eikelenboom P. The two faces of Alzheimer's disease. J Neurol 2000;247:500-5.

7 De Leeuw FE, de Groot JC, Bots ML, et al. Carotid atherosclerosis and cerebral white matter lesions in a population based magnetic resonance imaging study. J Neurol 2000;247:291-6

8 De Leeuw FE, De Groot JC, Oudkerk M, et al. Aortic atherosclerosis at middle age predicts cerebral white matter lesions in the elderly. Stroke 2000:31:425-9.

9 De Leeuw FE, de Groot JC, Oudkerk M, et al. Hypertension and cerebral white matter lesions in a prospective cohort study. Brain 2002;125:765-72.

10 Hofman A, OH A, Breteler MMB, et al. Atherosclerosis, apolipoprotein E, and prevalence of dementia and Alzheimer's disease in the Rotterdam Study. Lancet 1997:349:151-4.

11 Ott A, Breteler MM, de Bruyne MC, et al. Atrial fibrillation and dementia in a population-based study. The Rotterdam study. Stroke 1997;28:316-21.

12 Ott A, Stolk RP, van Harskamp F, et al. Diabetes mellitus and the risk of dementia: the Rotterdam study. Neurology 1999:53:1937-42.

13 van Popele NM, Grobbee DE, Bots ML, et al. Association between arterial stiffness and atherosclerosis: the Rotterdam study. Stroke 2001;32:454-60.

14 Folstein MF, Folstein SE, McHugh PR. "Mini-mental state." A practical method for grading the cognitive state of patients for the clinician. J Psychiatr Res 1975; 12:189-98.

15 Adams HP, Bendixen BH, Kappelle $\sqcup$, et al. Classification of subtype of acute ischemic stroke. Definitions for use in a multicenter clinical trial. TOAST. Trial of Org 10172 in Acute Stroke Treatment. Stroke 1993;24:35-41.

16 Vermeer SE, Den Heijer T, Koudstaal PJ, et al. Incidence and risk factors of silent brain infarcts in the population-based Rotterdam scan study. Stroke 2003;34:392-6.

17 van Zagten M, Kessels F, Boiten J, et al. Interobserver agreement in the assessment of cerebral atrophy on CT using bicaudate and Sylvian-fissure ratios. Neuroradiology 1999;41:261-4.

18 Scheltens P, Leys D, Barkhof F, et al. Atrophy of medial temporal lobes on MRI in "probable" Alzheimer's disease and normal ageing: diagnostic value and neuropsychological correlates. J Neurol Neurosurg Psychiatry 1992;55:967-72

19 Hansson L, Zanchetti A, Carruthers SG, et al. Effects of intensive bloodpressure lowering and low-dose aspirin in patients with hypertension: principal results of the Hypertension Optimal Treatment (HOT) randomised trial. HOT Study Group. Lancet 1998;351:1755-62.

20 Whitman GT, Tang Y, Lin A, et al. A prospective study of cerebral white matter abnormalities in older people with gait dysfunction. Neurology 2001;57:990-4.

21 Skoog I, Nilsson L, Palmertz B, et al. A population-based study of dementia in 85-year-olds. N Engl J Med 1993;328:153-8.

22 Shephard J, Blauw GJ, Murphy MB, et al. Pravastatin in elderly individuals at risk of vascular disease (PROSPER): a randomised clinical trial. Lancet 2002;360:1623-30.

23 De Groot JC, De Leeuw FE, Oudkerk $M$, et al. Periventricular cerebral white matter lesions predict rate of cognitive decline. Ann Neurol 2002;52:335-41.

24 De Groot JC, De Leeuw FE, Oudkerk M, et al. Cerebral white matter lesions and cognitive function: the Rotterdam scan study. Ann Neurol 2000;47:145-51

25 Filley CM. The behavioral neurology of cerebral white matter. Neurology 1998;50: 1535-40.

26 Scheltens $\mathbf{P}$, Barkhof $F$, Valk J, et al. White matter lesions on magnetic resonance imaging in clinically diagnosed Alzheimer's disease. Evidence for heterogeneity. Brain 1992;115:735-48.

27 Mann DM, Yates PO, Marcyniuk B. Some morphometric observations on the cerebral cortex and hippocampus in presenile Alzheimer's disease, senile dementia of Alzheimer type and Down's syndrome in middle age. J Neurol Sci 1985:69:139-59.

28 Sullivan EV, Shear PK, Mathalon DH, et al. Greater abnormalities of brain cerebrospinal fluid volumes in younger than in older patients with Alzheimer's disease. Arch Neurol 1993;50:359-73.

29 Nagy Z, Esiri MM, Jobst KA, et al. The effects of additional pathology on the cognitive deficit in Alzheimer disease. J Neuropathol Exp Neurol 1997; 56: 165-70.

30 Forette F, Seux ML, Staessen JA, et al. Prevention of dementia in randomised double-blind placebo-controlled systolic hypertension in Europe (Syst-Eur) trial. Lancet 1998;352:1347-51. 